Rakenteiden Mekaniikka (Journal of Structural Mechanics)

Vol. 50, No 3, 2017, pp. 309-312

https://rakenteidenmekaniikka.journal.fi/index

https:/doi.org/10.23998/rm.64857

(c)Author(s) 2017.

Open access under CC BY-SA 4.0 license.

\title{
Initial rotational stiffness of tubular joints with axial force in chord
}

\author{
Marsel Garifullin ${ }^{1}$, Sami Pajunen, Kristo Mela and Markku Heinisuo
}

\begin{abstract}
Summary. In the frame analysis, the local model of the joint must follow the behavior of the joint. When completing the elastic global analysis, the initial rotational stiffness of joints should be known to obtain reliable moment distributions in frames. This paper consists of two parts. The first one evaluates the existing calculation approach for the initial rotational stiffness of welded rectangular hollow section $\mathrm{T}$ joints. The validation with the experimental data shows that the current approach significantly underestimates the initial rotational stiffness. An improvement for determining the initial stiffness of $\mathrm{T}$ joints is proposed. The second part deals with the influence of the axial force in the chord on the rotational stiffness of the joint. The conducted numerical study shows the extreme reduction of the initial stiffness, when the chord is loaded by axial loads. To consider this effect in the frame analysis, the paper proposes a chord stress function for the initial rotational stiffness for square hollow section T joints, using the curve fitting technique.
\end{abstract}

Key words: rotational stiffness, hollow section joint, chord stress function, axial force

Received 14 June 2017. Accepted 15 August 2017. Published online 21 August 2017.

\section{Introduction}

Tubular structures cover a large range of applications, such as bridges, lattice masts, trusses. Welded joints are used in these constructions, leading to nice appearance and high stiffness and resistance. When completing the elastic global analysis, the initial rotational stiffness of joints should be known to obtain the reliable moment distribution between the members of the frame.

The pioneering research on tubular joints was conducted in [8]. After that, the extensive studies were undertaken on moment-loaded rectangular hollow section (RHS) joints by Tabuchi et al. [7], Szlendak [6], Packer [5], Yu [10] and other researchers [4, 11]. Currently, the design rules for moment resistance of $\mathrm{T}$ joints are contained in EN 1993-1-8:2005 [1].

The calculation methods for the rotational stiffness of welded rectangular hollow section $\mathrm{T}$ joints are based on the component method and presented in [3] and [9].

\footnotetext{
${ }^{1}$ Corresponding author. marsel.garifullin@tut.fi
} 
However, in contrast to the resistance, the initial stiffness rules have been validated with the very limited amount of experimental data, often leading to conservative results.

The focus of this paper is on the local initial rotational stiffness of the welded RHS T joint loaded by the in-plane bending moment. The welded $\mathrm{T}$ joint is comprised of a member (brace) connected in 90 degrees to the main member (chord). Fig. 1a illustrates the RHS T joint loaded with the in-plane moment $M$ in the end of the brace and by the axial force $N_{0}$ in the chord. On the figure, $b_{0}, h_{0}, t_{0}$ and $b_{1}, h_{1}, t_{1}$ are the geometrical dimensions of the chord and the brace; while $f_{y 0}$ and $f_{y 1}$ are respectively their yield strengths. The relative parameters are the brace-to-chord width ratio, $\beta=b_{1} / b_{0}$, and the chord width-to-thickness ratio, $\gamma=b_{0} / 2 t_{0}$.

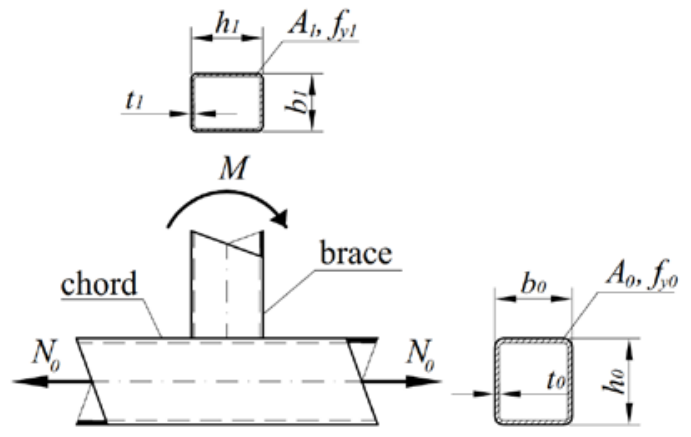

a)

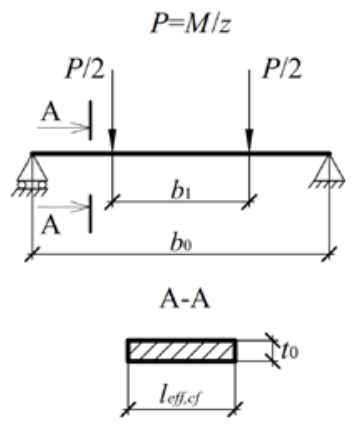

b)

Fig. 1. a) Rectangular hollow section T joint; b) beam model for component $k_{c f}$.

The first section evaluates the current calculation approach for the initial rotational stiffness of $\mathrm{T}$ joints and proposes the improved stiffness equation for one of the components. Section 2 studies the effect of the axial force in the chord on the initial rotational stiffness and proposes the corresponding chord stress function. The paper considers only the joints with $\beta \leq 0.85$.

\section{Initial rotational stiffness of $\mathrm{T}$ joints}

The initial rotational stiffness of the RHS T joint, $S_{j, \text { inin }}$, is determined similar to the stiffness of open section joints provided in EN 1993-1-8:2005 [1]:

$$
S_{j, i n i}=E z^{2} / \sum_{i} \frac{1}{k_{i}}
$$

where $E$ is the Young's modulus, $z$ is the lever arm $\left(z=h_{1}\right), k_{i}$ is the stiffness coefficient of the basic joint component $i$. The stiffness coefficients and their equations are provided in [3]. Normally, $k_{c f}$, the coefficient for the deformation of the chord flange, is the smallest when $\beta \leq 0.85$, contributing most to the local deformation of the joint. According to [3], $k_{c f}$ is calculated using the Euler-Bernoulli beam theory (Fig. 1b):

$$
k_{c f}=\frac{8 t_{0}^{3} l_{\text {eff }, c f}}{(1-\beta)^{3} b_{0}^{3}} \cdot \frac{1}{2+6 \beta /(1-\beta)},
$$

where $l_{\text {eff,cf }}$ is the effective length of the brace [3]:

$$
l_{\text {eff }, c f}=0.5 h_{1}+b_{0} \sqrt{1-\beta} \text {. }
$$


After the validation of the calculation approach with the experimental data, it was found that Eq. (2) significantly underestimates the initial rotational stiffness, with the theoretical values $S_{j \text {,ini }}$ accounting to $30-40 \%$ of the experimental ones, $S_{j, \text { ini,exp }}$. To provide a more accurate solution, the following equation was proposed:

$$
k_{c f}=\frac{20 t_{0}^{3} l_{e f f, c f}}{(1-\beta)^{3} b_{0}^{3}} \cdot \frac{1}{2+6 \beta /(1-\beta)} .
$$

Compared to the experimental results, Eq. (4) provides considerably more accurate values, with the $S_{j, \text { ini }} / S_{j, \text { ini,exp }}$ ratio close to one.

\section{Chord stress function for initial rotational stiffness of RHS T joints}

The axial forces acting in the chord are known to reduce the resistance of the joint [8]. This reduction is defined by the so-called chord stress functions, which are presented in EN 1993-1-8:2005. The similar phenomenon can be also observed for the initial rotational stiffness. In the global analysis, this can lead to the noticeable redistribution of moments in the members of trusses or frames, making the results of the analysis unreliable. This justifies the necessity to develop the chord stress function for the initial stiffness of joints. For that reason, Eq. (1) was presented in the following way:

$$
S_{j, i n i}=k_{s n, i p} E z^{2} / \sum_{i}\left(1 / k_{i}\right) \text {, }
$$

where $k_{s n, i p}$ is the chord stress function for the initial rotational stiffness.

The chord stress function was developed based on the 3D finite element analysis (FEM) for square hollow section T joints. The scope of the study was selected so cover the whole area of the practical interest and meet the requirements of EN 1993-1-8:2005. The numerical analyses were performed using the FE model employed in [2]. It was found that the compressive axial forces in the chord can decrease the stiffness of the joints by $50 \%$; while the tensile ones, controversially, can increase it to 30\%. Moreover, the observed effect was found to depend on $\beta$ and $\gamma$. Based on the obtained FEM results, the chord stress function was developed using the curve-fitting technique. The final function is presented in Eq. (6):

$$
k_{s n, i p}=\left\{\begin{array}{l} 
\begin{cases}1+0.001\left(1+1.7 \beta-2.6 \beta^{2}\right) n \gamma^{2}-2.7(|n|-0.8)^{2}, & -0.99 \leq n<-0.8 \\
1+0.001\left(1+1.7 \beta-2.6 \beta^{2}\right) n \gamma^{2}, & -0.8<n<0.8 \\
1+0.001\left(1+1.7 \beta-2.6 \beta^{2}\right) n \gamma^{2}-3.1(n-0.8)^{2}, & 0.8<n \leq 0.99\end{cases} \\
0.85<\beta<1.0: \text { linear interpolation between } \beta=0.85 \text { and } \beta=1.0
\end{array}\right.
$$

In Eq. (6), $n$ is the relative normal stress in the chord:

$$
n=\sigma_{0} / f_{y 0}=N_{0} / A_{0} f_{y 0}
$$

where $A_{0}$ is the cross-sectional area of the chord and $N_{0}$ is the axial load in the chord. A negative $n$ means compression, while a positive one indicates tension. 


\section{Conclusions}

This article analyzes the approach for the calculation the initial rotational stiffness of welded RHS $\mathrm{T}$ joints. Comparison with the experimental data shows that the original approach considerably underestimates the initial stiffness of joints with $\beta \leq 0.85$. For more accurate results, the improved equation is proposed for one of the components.

Based on the FEM analyses, axial forces in the chord were found to affect the initial rotational stiffness of square hollow section $\mathrm{T}$ joints. To avoid unreliable results in frame analyses, the chord stress function for the initial rotational stiffness of $\mathrm{T}$ joints was proposed, similar to that for the moment resistance. In the considered range, the proposed solution matches well to the numerical results and can be recommended for using in the frame design of RHS members.

\section{References}

[1] European Committee for Standardization (CEN). Eurocode 3. Design of steel structures, Part 1-8: Design of joints (EN 1993-1-8:2005). Brussels, 2005.

[2] M. Garifullin, M. Bronzova, T. Jokinen, M. Heinisuo and B. Kovačič. Effect of Fillet Welds on Initial Rotational Stiffness of Welded Tubular Joints. Procedia Engineering, 165:1643-1650, 2016. https://doi.org/10.1016/j.proeng.2016.11.905.

[3] D. Grotmann and G. Sedlacek. Rotational stiffness of welded RHS beam-to-column joints. Cidect 5BB-8/98. RWTH-Aachen, Aachen, 1998.

[4] L. Lu. The static strength of I-beam to rectangular hollow section column connections. Doctoral Dissertation. Delft University Press, Delft, 1997.

[5] J.A. Packer. Moment connections between rectangular hollow sections. Journal of Constructional Steel Research, 1-2(25):63-81, 1993. https://doi.org/10.1016/0143-974X(93)90052-T.

[6] J. Szlendak. Beam-column welded RHS connections. Thin-Walled Structures, 1(12):63-80, 1991. https://doi.org/10.1016/0263-8231(91)90027-G.

[7] M. Tabuchi, H. Kanatani and T. Kamba. The local strength of welded RHS T joints subjected to bending moment. CIDECT report 5AF-84/5E, 1984.

[8] J. Wardenier. Hollow Section Joints. Delft University of Technology, Delft, 1982.

[9] K. Weynand, J.-P. Jaspart, J.-F. Demonceau and L. Zhang. Component method for tubular joints. CIDECT Report 16F - 3/15, 2015.

[10] Y. Yu. The static strength of uniplanar and multiplanar connections in rectangular hollow sections. Doctoral Dissertation. Delft University of Technology, Delft, 1997.

[11] X.L. Zhao. Deformation limit and ultimate strength of welded T-joints in coldformed RHS sections. Journal of Constructional Steel Research, 2(53):149-165, 2000. https://doi.org/10.1016/S0143-974X(99)00063-2.

Marsel Garifullin, Sami Pajunen, Kristo Mela, Markku Heinisuo

Tampere University of Technology

P.O. Box 600, FI-33101 Tampere, Finland

marsel.garifullin@tut.fi sami.pajunen@tut.fi kristo.mela@tut.fi markku.heinisuo@tut.fi 